\title{
Effectiveness of a physiotherapy-initiated telephone triage of orthopedic waitlist patients
}

\author{
This article was published in the following Dove Press journal: \\ Patient Related Outcome Measures \\ 19 August 2011 \\ Number of times this article has been viewed
}

\author{
Joanne Morris' \\ Karen Grimmer-Somers ${ }^{2}$ \\ Saravana Kumar ${ }^{2}$ \\ Karen Murphy ${ }^{3}$ \\ Lisa Gilmore' \\ Bryan Ashman' \\ Chandima Perera \\ Kathryn Vine' \\ Corinne Coulter ${ }^{1}$ \\ 'The Canberra Hospital, ACT \\ Government Health Directorate, \\ Canberra, ACT, Australia; \\ ${ }^{2}$ International Centre for Allied \\ Health Evidence, University of South \\ Australia, Adelaide, SA, Australia; \\ ${ }^{3}$ ACT Government Health \\ Directorate, Canberra, \\ ACT, Australia
}

Correspondence: Karen Grimmer-Somers International Centre for Allied Health Evidence (iCAHE), University of South Australia, City East Campus, GPO Box 247I, Adelaide, SA 5000, Australia

Tel +61883022769

Fax +6I 883022766

Email karen.grimmer-somers@unisa. edu.au
Background: There is generally a lengthy wait on outpatient orthopedic waiting lists in Australian public hospitals to consult a specialist. Patients then wait again for surgery, if required. Patients with higher need are rarely prioritized, and there is the potential for increased morbidity for those who wait. There is generally no option of alternative care whilst waiting. This paper compares historical orthopedic outpatient clinic data with the outcomes of a physiotherapy-led initiative in one large Australian tertiary hospital.

Methods: Two physiotherapists working within-scope conducted a telephone triage (October to December 2010) using a standard instrument for all new patients on the orthopedic waiting list. They were offered primary treatment options of retaining their appointment, being discharged, referral to a new model of assessment (multidisciplinary specialist clinic), or referral to physiotherapy. The outcomes were costs of the service, waiting time, and percentage of patients taking up management options. This was compared with a historical sample of new patients on the orthopedic waiting list (January to March 2009), whose treatment consumption was tracked longitudinally.

Results: The telephone triage resulted in $16.4 \%$ patients being discharged directly (compared with $0.1 \%$ comparison sample). For approximately AU\$17.00 per patient, the telephone triage process released 21 booked appointments on the outpatient clinic waiting list. Moreover, approximately $26 \%$ patients were referred directly to physiotherapy, which was not a primary management option in the comparison sample. The waiting time for an appointment, for those patients who remained on the waiting list, was significantly shorter for the telephone triage sample than the comparison sample. There were significantly higher rates of failure to attend appointments, and significantly lower rates of discharge, in the comparison sample, than the telephone triage sample.

Conclusion: A physiotherapist-led intervention offering alternative management options whilst patients waited for an orthopedic outpatient clinic consultation appears to be cost-effective, and patient-centered.

Keywords: extended scope practice, orthopedics, evaluation

\section{Background}

The number of people on waiting lists for Australian public hospital orthopedic outpatient clinics, and the length of time they wait, has steadily increased over recent years. ${ }^{1}$ The increase relates to factors such as the size of public hospital budgets and their allocation to outpatient services compared with the number of public patients seeking attention, the increase in prevalence of joint disease such as osteoarthritis and rheumatoid arthritis, the affordability of private health insurance, and the availability of private specialist care. ${ }^{2,3}$ The increase also relates to imbalances between the available 
number of orthopedic and rheumatology specialists, the hours they are able to work in the public system, and the complexity of orthopedic problems affecting the generally aging population. ${ }^{4}$ Typically, patients are referred to public hospital orthopedic waiting lists by their general medical practitioners or from other outpatient clinics. The validity of the referral is rarely assessed, and once on the waiting list, patients' needs are rarely reviewed, nor are they offered alternative treatment as an interim measure. Thus patients may wait for several years for an appointment which may in fact not be necessary, and then they wait again if surgery is scheduled. Moreover, without interim assessment and advice whilst waiting, their physical function and community independence is likely to deteriorate. ${ }^{5}$

Extending the scope of physiotherapy practice has been proposed as one way to deal with long public hospital waiting lists in Australia. ${ }^{6}$ Extended scope orthopedic physiotherapy has been widely practiced in the UK for the past decade, and has been shown to be an effective substitute for public sector medical assessment and care, when medical services are unavailable or scant. ${ }^{7,8}$ Physiotherapists in orthopedic clinics who operate beyond conventional scope of practice have high level orthopedic clinical skills and additional specialist training in ordering and interpreting imaging, administering corticosteroid injections where clinically indicated, and prescribing. Thus they can offer patients alternative forms of management, which may attenuate morbidity associated with waiting, and reduce the waiting list to those patients who truly require an orthopedic appointment. Current research suggests that such interventions will potentially reduce the time that patients wait for an orthopedic appointment, and for subsequent surgery. ${ }^{9}$ In Australia, however, advanced/ extended scope orthopedic physiotherapy services have been introduced in a piecemeal fashion over the last 10 years, and the effectiveness of these mostly pilot interventions has been variably evaluated and reported in grey literature (eg, Osborne and colleagues, ${ }^{3}$ Moore, ${ }^{10}$ and Victorian Government Department of Human Services ${ }^{11}$ ).

This paper describes the outcomes of an Australian initiative in which physiotherapists working within-scope prospectively telephone-triaged patients on the public orthopedic waiting list at one large Australian tertiary hospital, and referred them into alternative management pathways whilst they waited. While the use of telephone triage to deal with staff shortages is not novel, ${ }^{12}$ and physiotherapists working within scope have been shown to effectively direct triage patients in an orthopedic clinic, ${ }^{13}$ there is little in the literature regarding the effectiveness of physiotherapists triaging orthopedic waiting list patients by telephone. Two alternative management pathways were offered in this project: physiotherapy and a fortnightly multidisciplinary team (MDT) clinic staffed by an extended scope physiotherapist (in training) working in conjunction with an orthopedic surgeon and a consultant rheumatologist.

The aim of this initiative was to increase patients' access to care, and to improve the timeliness of care appropriate to their current need. This ensured that the patients remaining on the waiting list for surgery were likely to truly require it, and that they would not wait as long as they would under usual circumstances.

\section{Method \\ Ethics approval}

Ethics approval was provided by the ACT Health Human Research Ethics Committee (Protocol ERHLR.10.259, approved December 15, 2010).

\section{Study design}

The study was quasi-experimental, comparing outcomes from a physiotherapy-led telephone triage of new patients on the orthopedic outpatient waiting list with those of a similar historical control group.

\section{Setting}

The study was carried out at The Canberra Hospital (TCH), which is the regional trauma center for the Australian Capital Territory (ACT) and provides trauma services to New South Wales (NSW) residents in the greater southern region of NSW Health. It has a catchment population of 500,000 and offers the only public referral point for orthopedic outpatients in the region. ${ }^{14}$ All patients attending this service must have a referral from a medical practitioner (eg, general practitioner (GP), sports physician, and other medical/surgical specialist). As with many public elective services in the ACT, patients referred to TCH Orthopedic Outpatient Department experience lengthy waiting times for appointments and any subsequent surgery.

\section{Terminology}

Working within physiotherapy scope refers to working within registerable and recognized physiotherapy competencies (see Figure 1). ${ }^{15}$ Physiotherapists working beyond usual scope refers to clinicians working at a high level within scope (advanced scope), or applying extended scope training in imaging, prescribing, and application of evidence in clinical decision-making. Recognition of physiotherapists' 


\section{Advanced scope of practice}

A role that is within currently recognised scope of practice for that profession, but that through custom and practice has been performed by other professions. The advanced role would require additional training, competency development as well as significant clinical experience and formal peer recognition. This role describes the depth of practice.

\section{Extended scope of practice}

A role that is outside the currently recognised scope of practice and requires legislative change. Extended scope of practice requires some method of credentialing following additional training, competency development and significant clinical experience. Examples include prescribing, injecting and surgery. This role describes the breadth of practice.

Figure I Current definitions of scope of practice from the Australian Health Workforce Advisory Committee. ${ }^{15}$

extended scope training in Australia is currently on an institutional level and not underpinned by formal accredited training programs. Extended scope practice is also limited by legislation.

\section{Triage intervention}

A telephone triage tool (Appendix 1) was developed specifically for this project by a multidisciplinary subgroup of the larger project steering committee, and tested for face, content, and construct validity prior to use with the institution's survey committee ${ }^{\mathrm{a}}$.

The telephone triage intervention was conducted in October-November 2010 for all consecutive new patients with an appointment for the orthopedic outpatient clinic (appointments scheduled from January 1 to April 30, 2011). These patients had been referred in general anticipation of nonurgent orthopedic opinion regarding surgical options for hip/knee joint replacement or for shoulder, elbow, wrist, ankle, and foot conditions.

Two HP3-level physiotherapists (working within-scope) delivered the tool over the telephone to new patients with a booked appointment on the orthopedic waiting list (described later). The physiotherapists had previously established their reliability of administration and interpretation of patient responses to the survey instrument. Their hourly cost

${ }^{a}$ The steering committee comprised two orthopedic surgeons, a consultant rheumatologist, an epidemiologist, four physiotherapists, two policy-makers, and one consumer representative. (including 35\% oncosts) approximated AU $\$ 51.45$ each, and each telephone interview took between 10 and 15 minutes. ${ }^{16}$ For cost calculations, the authors of this paper allowed 10 minutes per hour of "down time" where the physiotherapists called numbers for which there was no reply. Thus, in an average hour, four interviews could be conducted (approximate cost per interview AU\$12.86).

During the telephone triage, management options were offered, comprising (1) retaining the booked orthopedic clinic appointment, (2) cancelling the appointment if not required, (3) referral directly to conservative management (generally physiotherapy), or (4) referral directly to a new service. This was a fortnightly MDT clinic, which assessed patients with complex orthopedic conditions which could not be readily assessed by telephone alone.

The MDT clinic was conducted by an extended scope physiotherapist (in training) (hourly cost including oncosts AU \$58.03), an orthopedic surgeon, and a consultant rheumatologist (each with estimated hourly rates at the agreed TCH salaried specialist rate AU \$128.76) ${ }^{16}$ The physiotherapist had a relevant masters degree in musculoskeletal physiotherapy. The 3-hour clinic had the capacity to assess 18 patients (six each being assessed by the three health providers who then conferred at the end of the clinic). The MDT clinic had been initially conceived as a way of obtaining medical specialist validation of the activities of the extended scope physiotherapist (in training). The average cost of a patient consultation at the MDT (combined salaries of the three health professionals) was AU\$52.59. 
Historical baseline (comparison) data were provided by a retrospective audit, which extracted data from records of consecutive new orthopedic patients on the TCH orthopedic outpatient clinic waiting list between January 1 and March 31, 2009. Data were extracted in February 2011. Preliminary analysis of the volume of referrals onto the orthopedic waiting list indicated that there were no significant month-by-month variations in referrals at TCH, and thus 3 months' historical data would provide appropriate information on "usual waiting list" outcomes.

\section{Patient samples}

All data were accessed from the ACT Patient Information System and the Clinical Records Information System ${ }^{\mathrm{b}}$.

\section{Data management and analysis}

Considering the historical (comparison) audit data, all patients' records were individually tracked for primary outcomes (the first management option and its date). Primary management options were to proceed to the surgical waiting list, be rebooked to a subsequent orthopedic outpatient clinic, be referred to physiotherapy, or be discharged.

The telephone triage intervention offered primary management options of (1) keeping the booked appointment, (2) cancelling the booked appointment because patients no longer required it, (3) attending conservative management (mostly physiotherapy or podiatry) whilst retaining the booked appointment as a backup plan, or (4) attending the MDT clinic whilst retaining the booked outpatient appointment as a backup.

For those patients who attended either the MDT clinic or physiotherapy as primary management options, the next (secondary) management option was also recorded, which could be: (1) discharge with cancellation of the booked appointment, (2) refer for further investigations before a management decision was made, (3) trial of conservative treatment with a view to cancelling the booked appointment, and (4) trial of conservative treatment with a view to retaining the booked appointment.

For both samples, the number of patients who failed to attend, or who cancelled, or who rescheduled appointments, were reported. Flowcharts of management options were developed for "usual practice" (baseline sample) and the physiotherapy-led telephone triage. These are described in Figures 2 and 3.

${ }^{\mathrm{b}}$ These systems were only accessed by current ACT Government Health Directorate employees. Data were de-identified for collation purposes.

\section{Data analysis}

The samples were described for gender and age, and if known, median waiting times for an orthopedic clinic appointment, and for surgery (25th-75th percentiles). Non-parametric tests were conducted of differences between samples in waiting times using Mann-Whitney $U$ tests. The numbers (and percentages) of patients consuming different primary and secondary management options were reported and compared, using chi-square tests of proportions. Significance for all tests was set at $P<0.05$. Costs and cost differences between the telephone triage initiative and the comparison sample were considered.

\section{Results}

\section{Comparison sample}

There were 142 new outpatient appointments booked during the period of the comparison audit (which reflected $34 \%$ of all patients on the orthopedic outpatient waiting list at that time). Tracking these, 30 patients did not attend their appointment (20 failed to attend without explanation, and 10 cancelled because they had surgery elsewhere). The remaining 112 patients comprised 50.7\% males and 49.3\% females, with an average age of 51.2 years (standard deviation 19.6) (no gender-age differences). A total of $92 \%$ were referred by GPs.

The conversion rate to surgery at the time of the audit (February 2011) was $20.4 \%$, with a median time to receive surgery at TCH after the first orthopedic clinic appointment of 7.8 months (25th-75th percentiles 5.3-16.6 months). The median waiting time for patients to receive an orthopedic appointment was 25.1 months (25th-75th percentiles $24.3-$ 31.3 months). This does not take into account (the unknown number of) patients who make subsequent decisions to receive surgery elsewhere, or who remove themselves from the waiting list. See Figure 2 for details on the management options and the patient numbers.

\section{Telephone triage}

All 120 new patients with a scheduled orthopedic outpatient clinic appointment between January and April 2011 were telephoned, and the triage tool was administered to 116 . The remaining four patients could not be contacted despite three telephone calls at different times of the day. The contactable telephone triage sample reflected $32.6 \%$ of all patients on the waiting list at that time. There were similar gender proportions ( $49 \%$ males, $51 \%$ females), with a median age 65.1 years (25th-75th percentiles $51-74$ years). There were no gender-age differences. The primary management 


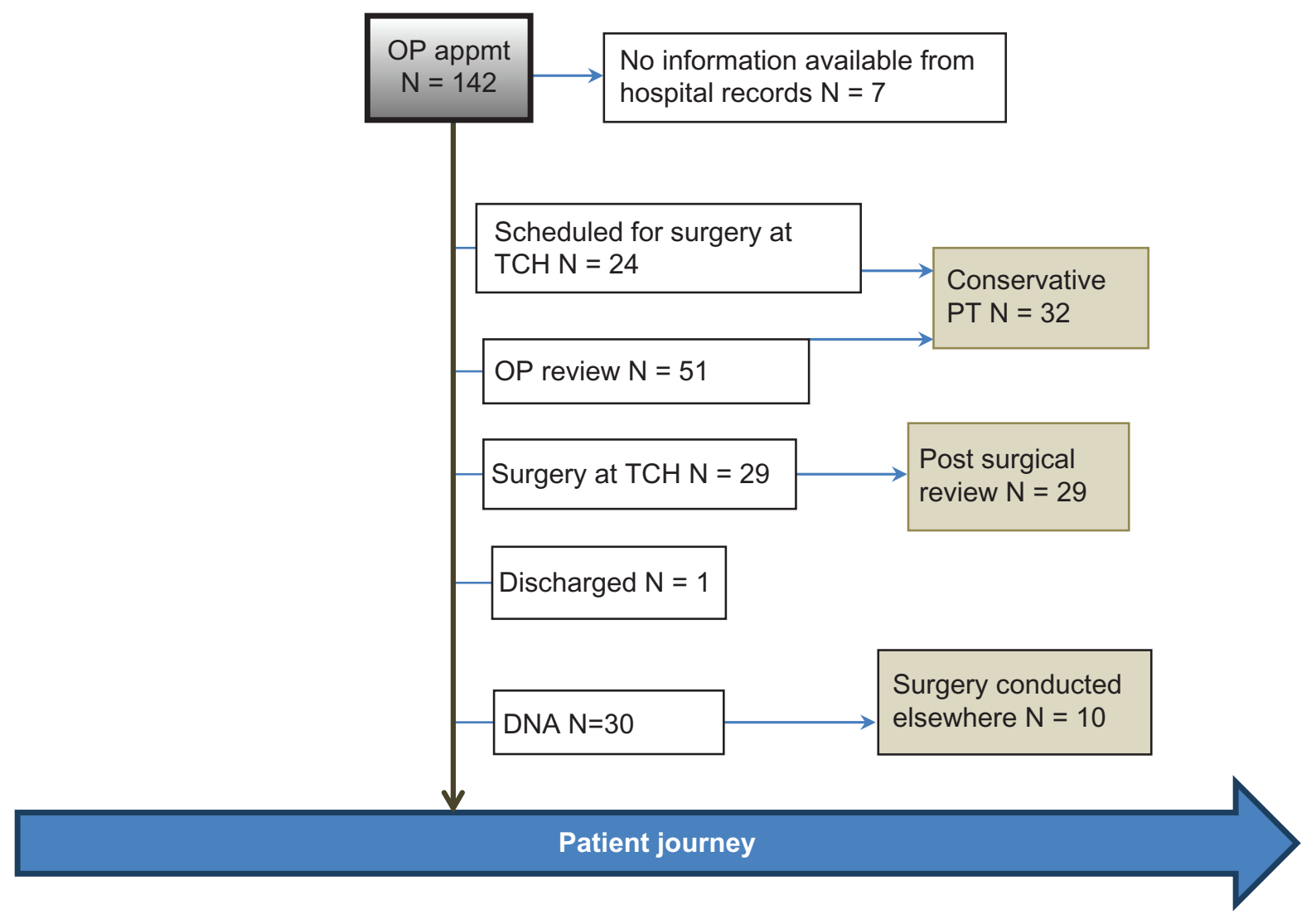

Figure 2 Usual processes of care and decision-making.

Abbreviations: DNA, did not attend; appmt, appointment; OP, outpatient; PT, physiotherapy; TCH, The Canberra Hospital.

outcomes for this sample were described earlier (this being the decision made over the telephone, not at subsequent clinic appointments).

The median waiting time from entering the waitlist to being triaged was 6.6 months $(25$ th -75 th percentiles $5.8-7.5$ months), and the median time between being triaged and booked for a surgical opinion was 3.5 months (25th-75th percentiles 2.7-4.3 months). See Figure 3 for details on the management options and patient numbers.

\section{Comparison between samples}

There was no significant difference between samples in gender or age. The telephone triage sample had a significantly shorter waiting time for an orthopedic clinic appointment than the control sample $(P<0.05)$. A comparison of the primary management outcomes of the samples is provided in Table 1.

The most obvious effect of the telephone triage was the high percentage of patients who were discharged without an immediate or ongoing need for further orthopedic outpatient appointments. This outcome was obtained at the initial telephone engagement $(\mathrm{N}=19)$ and again following attendance at the MDT clinic $(\mathrm{N}=2)$. The physiotherapy discharged patients are not considered in this analysis as it is not known how many consultations they had with the physiotherapist to arrive at the point of discharge. Using the costs of the physiotherapy-led telephone triage, the patients discharged over the telephone cost an estimated total of AU\$244. The patients discharged after one MDT consultation $(\mathrm{N}=2)$ cost a total of AU $\$ 105.18$. The discharge decision was discussed with, and agreed by, patients. Thus, for a total cost of approximately AU $\$ 350$, a total of 21 booked appointments were released on the outpatient clinic waiting list due to this initiative (costing approximately AU $\$ 17.00$ per patient).

Had these patients proceeded to an outpatient clinic appointment with a specialist orthopedic surgeon or a consultant rheumatologist, the appointment would have cost a total of AU\$675.99 (based on an estimated 15 minutes per outpatient appointment). Thus, not only were 21 potential outpatient clinic appointments released for this sample of 116 patients (18\%) (who under "usual practices" would have proceeded to a clinic appointment), but there were cost savings of approximately $48 \%$ in the discharge process. 


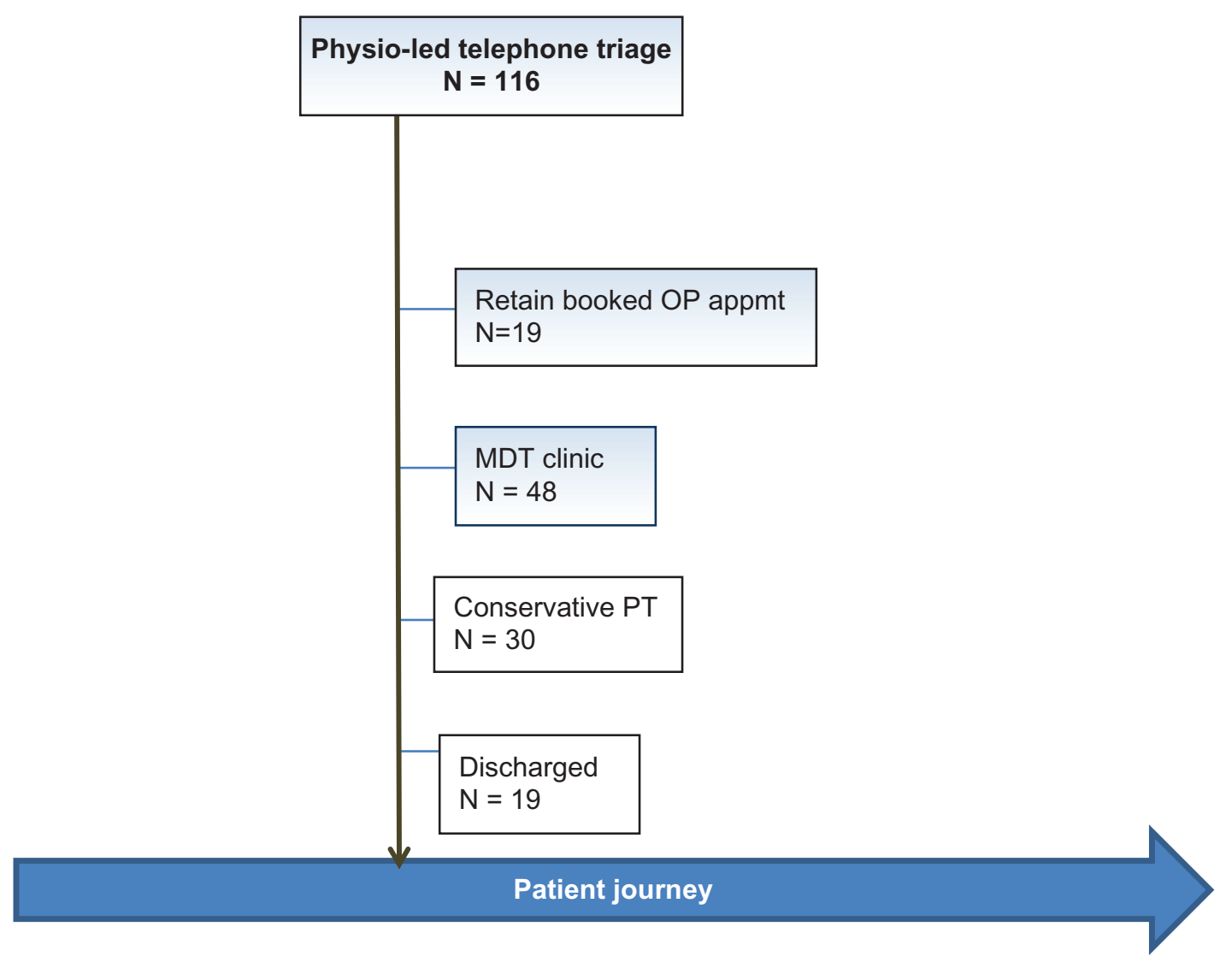

Figure 3 Physiotherapy-led processes of care and decision-making.

Abbreviations: appmt, appointment; MDT, multidisciplinary team; OP, outpatient; PT, physiotherapy.

There was also a significantly higher percentage of referrals to physiotherapy in the telephone triage sample (referred directly or via the MDT clinic), compared with the historical sample. The outcomes of these management options would need to be reviewed in a future audit to determine whether future surgical intervention was provided to these patients. Although the percentage of patients proceeding to surgery was similar in the two samples, the median waiting time for those patients who remained on the orthopedic outpatient waiting list was significantly shorter for the telephone triage sample than the baseline sample. There was a considerably higher rate of failure to attend appointments in the baseline sample than the telephone triage sample, which may have reflected patients who did not believe they needed the appointment, or who had gone elsewhere for treatment and failed to notify the hospital.

Considering the secondary outcomes of the telephone triage sample (outcomes of those patients who were referred to MDT or physiotherapy), of those patients who were referred to the MDT clinic ( $\mathrm{N}=48), 26(54.2 \%)$ were advised to retain their appointment on the outpatient waiting list with a view to proceeding to surgery, seven were referred for further investigations and then to return to the MDT clinic for a final decision on management (14.6\%), four were referred to physiotherapy with a view to cancelling their outpatient appointment (8.3\%), two were discharged (4.2\%), seven failed to attend the MDT

Table I Comparison of primary outcomes

\begin{tabular}{|c|c|c|}
\hline Primary outcome & $\begin{array}{l}\text { Baseline audit } \\
N=142\end{array}$ & $\begin{array}{l}\text { Telephone } \\
\text { triage initiative } \\
N=116\end{array}$ \\
\hline No available information & $7(0.5 \%)$ & $0(0.0 \%)$ \\
\hline Discharged & I (0.I\%) & $19(16.4 \%)$ \\
\hline Received surgery at $\mathrm{TCH}$ & $29(20.4 \%)$ & Not an option \\
\hline Scheduled for surgery at $\mathrm{TCH}$ & $24(16.9 \%)$ & Not an option \\
\hline $\begin{array}{l}\text { Retained booked OP } \\
\text { appointment with a view to } \\
\text { proceeding to surgery }\end{array}$ & 51 (35.9\%) & $19(16.4 \%)$ \\
\hline DNA & $30(21.1 \%)$ & Not an option \\
\hline Referred to MDT & Not an option & 48 (4I.4\%) \\
\hline Referred to physiotherapy & $\begin{array}{l}\text { Not a primary option } \\
\text { (could occur as a } \\
\text { secondary option, } \\
\text { see Figure IA) }\end{array}$ & $30(25.9 \%)$ \\
\hline
\end{tabular}

Abbreviations: OP, outpatient; MDT, multidisciplinary team; $\mathrm{TCH}$, The Canberra Hospital. 
appointment (14.6\%), and $10(20.8 \%)$ cancelled their appointment (and thus opted to remain on the outpatient appointment waiting list).

Of those patients who were directed during the telephone triage to physiotherapy $(\mathrm{N}=30)$, seven $(23.3 \%)$ were advised to remain on the waiting list on track for surgery (with or without physiotherapy), one was discharged (3.3\%), six continued with physiotherapy treatment with a view to cancelling their booked outpatient clinic appointment (20.0\%), and eight failed to attend (26.7\%). The general waiting time for commencement of physiotherapy was 2-3 weeks after telephone triage. In every instance, a letter was sent to the referring doctor outlining the management plans, and recommending that the doctor monitor patient progress.

The secondary outcomes from the telephone triage are reported in column 1 in Table 2.

Thus, considering the combined primary and secondary outcomes of the contactable telephone triage sample, 52 patients $(44.8 \%)$ were on a scheduled track for surgery by retaining their orthopedic outpatient appointments, 32 (27.6\%) patients were discharged (or likely to be discharged) as a result of the telephone triage directly, or after attendance at MDT or physiotherapy. There were 15 failures to attend either MDT or physiotherapy (19.2\%). As DNA was not a primary management option for the telephone triage, this number is deflated by division by the total number in the telephone triage, when considering primary and secondary outcomes. The combined primary and secondary outcomes of the new initiative are reported in the second column of Table 2.

Considering both samples and the available primary and secondary outcome data, the main differences between the samples relating to the new initiative were:

Table 2 Key secondary outcomes and combined primary and secondary outcomes for the telephone triage sample

\begin{tabular}{|c|c|c|}
\hline Outcome & $\begin{array}{l}\text { Secondary } \\
\text { outcomes } \\
N=78\end{array}$ & $\begin{array}{l}\text { Combined primary } \\
\text { and secondary } \\
\text { outcomes } \\
\mathrm{N}=1 \mathrm{l} 6\end{array}$ \\
\hline $\begin{array}{l}\text { Discharged or likely } \\
\text { to be discharged }\end{array}$ & 13 (16.7\%) & 32 (27.6\%) \\
\hline $\begin{array}{l}\text { Retained booked OP } \\
\text { appointment with a view } \\
\text { to proceeding to surgery }\end{array}$ & 33 (42.3\%) & 52 (44.8\%) \\
\hline DNA & I5 (19.2\%) & 15 (I2.9\%) \\
\hline $\begin{array}{l}\text { Referred to, or reviewed } \\
\text { in, MDT }\end{array}$ & $8(10.3 \%)$ & $56(48.3 \%)$ \\
\hline
\end{tabular}

Abbreviations: OP, outpatient; MDT, multidisciplinary team.
- A nonsignificant increase in the number of people who might proceed to surgery $(P>0.05)$,

- A significant increase $(P<0.05)$ in the number of people who could be discharged (and not require an outpatient appointment at all). There were cost savings in achieving discharge before consulting a specialist at the orthopedic outpatient clinic.

- A nonsignificant increase $(P>0.05)$ in the number of people who were referred to physiotherapy as a management option, and

- A significant decrease $(P<0.05)$ in the patients who did not attend their appointment.

The key outcomes for comparison between the samples are provided in Figure 4.

\section{Discussion}

Using samples that were comparable in terms of demographics and size, this study reports on a rare opportunity in Australia to consider changes which could be made to a lengthy orthopedic outpatient waiting list by a physiotherapy-led telephone triage initiative. This process used a standard set of questions, and referred patients to alternative interim management options whilst they waited for an orthopedic appointment. Thus, access to appropriate care and the timing of care were improved by one low-cost intervention. The authors' findings mirrored those from other research..$^{6-8,13}$

\section{Considering progression to surgery}

Compared with baseline data, the physiotherapy-led initiative appeared to validate the need for referral for surgery for at least $40 \%$ of patients on the waiting list. It also identified a significant number of patients who were unlikely to require surgery and could be discharged from the waiting list, thus releasing orthopedic surgeons' time and providing opportunities for patients who really did need surgery to be assessed sooner. This initiative also decreased the number of appointments which patients failed to attend, possibly because the contact with the hospital had reinforced their attendance, and/or their interest in retaining their appointment. This also suggested that budget forecasting for orthopedic surgeons' time to consult in TCH outpatient clinic, and to subsequently perform their surgery, was potentially inflated by approximately $40 \%$.

It is not possible to compare the samples in terms of waiting times for surgery, because for the telephone triage sample, surgery was unlikely to have occurred at the time of data collation. Follow-up is required to determine whether all 


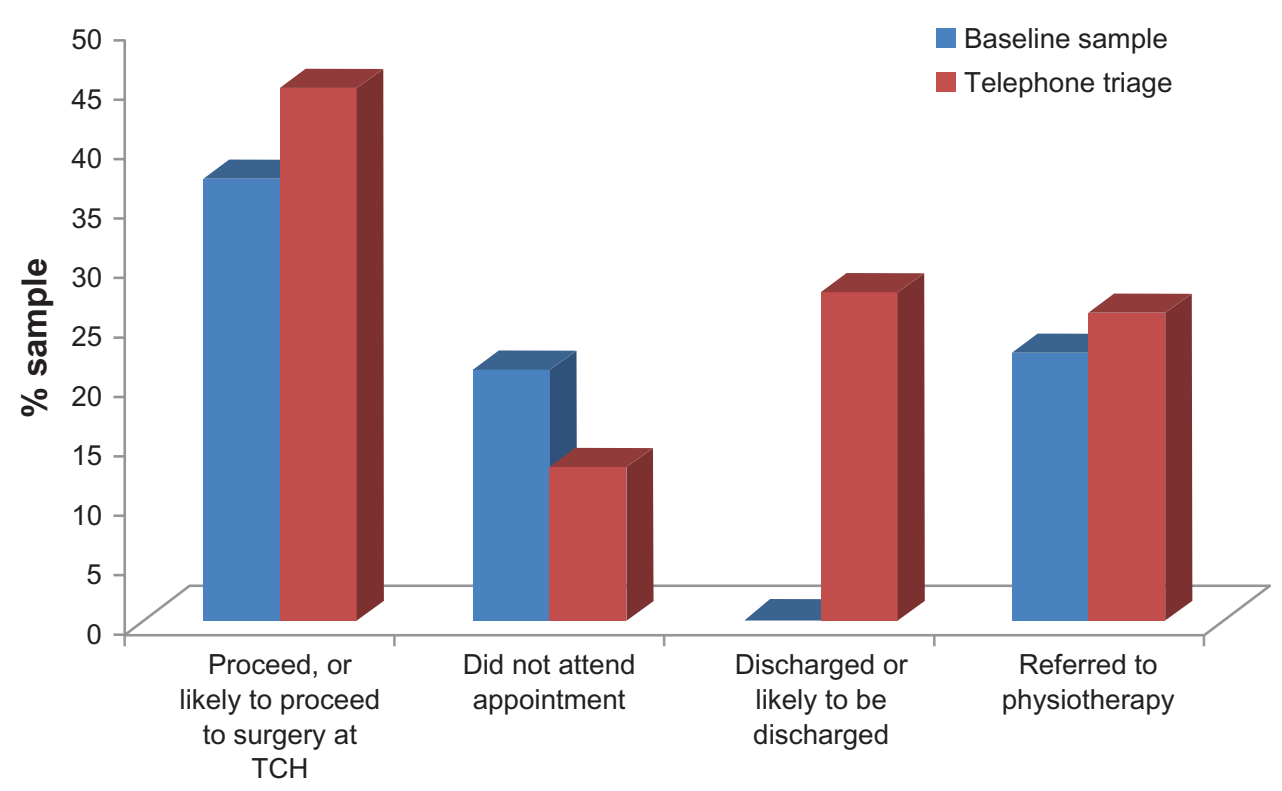

Figure 4 Key combined (primary and secondary) outcomes between the samples. Abbreviation: $\mathrm{TCH}$, The Canberra Hospital.

patients who retained their booked outpatient appointment as a result of one of the management options from the telephone triage did in fact proceed to surgery, and to test whether those patients who cancelled their appointment proceeded to surgery at a later date.

\section{Considering the MDT clinic}

The MDT was initially conceived as a way of validating the skills of the extended scope physiotherapist in-training, by having their assessments and decisions overviewed by two medical specialists. It is possible that the extended-scope physiotherapist will act alone in the future by leading more clinics, and only consulting with the medical specialists on difficult/complex patients when a clear management pathway is not obvious. Based on the current rate of consulting six patients per 3-hour MDT clinic, this could decrease the costs per patient attending this clinic to AU\$29 (based on the HP4-level physiotherapist wage including oncosts) (compared with the current per-patient cost of AU\$52.59). Notwithstanding the potential for a cheaper service, the benefits of the MDT are the multidisciplinary opportunities, which have the potential to improve outcomes for patients with complex problems. This clinic has highlighted the benefits of multidisciplinary teamwork, and has not only provided patients with access to broad specialist decisions but has also provided a learning platform for all health professionals involved. The MDT clinic may well provide training opportunities in the future for other extended scope physiotherapists, and also for medical trainees (students, interns, and registrars), as it offers a rare opportunity to showcase how different professionals consider complex orthopedic cases. An MDT therefore may well become a primary management option for orthopedic outpatients in the future. The findings from this study are supported by a recent Canadian report, which suggests that innovative teams working together and separately are required to address the challenges of waiting lists and chronicity of conditions. ${ }^{17}$

\section{Considering referral to physiotherapy}

Physiotherapy was only historically available as a secondary management option (after an orthopedic clinic appointment). Not every patient received a physiotherapy referral, and nothing could be tracked about the appropriateness of the referral process. From the new initiative, physiotherapy was offered as a primary management option, with or without a view to proceeding to a surgical opinion. The perceived benefits of referral to physiotherapy could be inferred by the number of patients who were advised to trial a course of physiotherapy with a view to cancelling their booked outpatient clinic appointment (12.8\%), either from the MDT clinic or the physiotherapy clinic. In the baseline sample, $22.5 \%$ patients were referred to physiotherapy as a secondary management option after having waited a long time for an outpatient appointment. One could question whether these patients in fact could have been directed to physiotherapy as primary management strategy, without waiting as long as they did, or even consuming an orthopedic outpatient 
appointment which could have been used by someone in greater need. ${ }^{6,7}$

\section{Conclusion}

Physiotherapists telephone-triaging patients with an orthopedic outpatient appointment can assist in the reorganization and prioritization of a lengthy orthopedic outpatient waiting list. This offers a low cost, high return option to an institution with concerns about managing its orthopedic waiting list. The high returns are in terms of cost savings (particularly orthopedic surgeons' time being spent on patients who truly require a surgical opinion), and societal benefits such as improvements in patients' access to timely and appropriate care, which will potentially decrease morbidity.

\section{Disclosure}

The authors report no conflicts of interest in this work.

\section{References}

1. Australian Institute of Health and Welfare. Australian Hospital Statistics 2003-2004, AIHW cat. No. HSE 37. Health Services Series no. 23. Canberra: Australian Government; 2005.

2. Ostendorf M, Buskens E, van Stel H, et al. Waiting for total hip arthroplasty: avoidable loss in quality time and preventable deterioration. J Arthroplasty. 2004;19(3):302-309.

3. Osborne R, Haynes K, Jones C, Chubb P, Robbins D, Graves S. Orthopaedic Waiting List Project: summary report. Victoria: Department of Human Services, State of Victoria; 2006. Available from: http://www. health.vic.gov.au/surgery/pubs/owlsumrep.pdf. Accessed June 22, 2011.

4. Borland J. Report to the Australian Competition and Consumer Commission on: Australian Medical Workforce Advisory Committee The Orthopaedic Surgery Workforce in Australia, an Update: 1998 to 2009. Victoria: Department of Economics, University of Melbourne; 2001. Available from: http://www.accc.gov.au/content/item.phtml?item $\mathrm{Id}=308230$ \&nodeId=ef78fecd $69 \mathrm{c} 6 \mathrm{dc} 051 \mathrm{cc} 684 \mathrm{c} 310 \mathrm{e} 10 \mathrm{fde} \& \mathrm{fn}=$ Repor $\mathrm{t} \% 20$ to $\% 20$ ACCC $\% 20-\% 20$ AMWAC $\% 20$ Orthopaedic\%20Surgery $\% 20$ Workforce\%20 in\%20 Australia.pdf. Accessed June 22, 2011.

5. Löfvendahl S, Eckerlund I, Hansagi H, Malmqvist B, Resch S, Hanning M. Waiting for orthopaedic surgery: factors associated with waiting times and patients' opinion. Int J Qual Health Care. 2005;17(2):133-140.
6. Oldmeadow LB, Bedi HS, Burch HT, Smith JS, Leahy ES, Goldwasser M. Experienced physiotherapists as gatekeepers to hospital orthopaedic outpatient care. Med J Aust. 2007;186(12):625-628.

7. Hattam P. The effectiveness of orthopaedic triage by extended scope physiotherapists. Clin Governance. 2004;9(4):244-252.

8. Kotecha A, Meyer C, Crichlow T, Kakati S. Should extended scope physiotherapists place patients directly on arthroscopy lists? J Bone Joint Surg Br. 2010;92-B Suppl 2:322-323.

9. McPherson K, Kersten P, George S, et al. A systematic review of evidence about extended roles for allied health professionals. $J$ Health Serv Res Policy. 2006;11:240-247.

10. Moore B. Extended scope practitioners: our changing role in rural practice. Proceedings of the 9th National Rural Health Conference; 2007. http://9thnrhc.ruralhealth.org.au/program/docs/papers/moore_D3.pdf. Accessed June 22, 2011.

11. Victorian Government Department of Human Services. Better skills, best care - stage 1. Final report 2007. Melbourne: State Government of Victoria; 2007.

12. Letourneau MA, MacGregor DL, Dick PT, et al. Use of a telephone nursing line in a paediatric neurology clinic: one approach to the shortage of specialists. Pediatrics. 2003;112:1083-1087.

13. MacKay C, David AM, Mahomed N, Badley EM. Expanding roles in orthopaedic care: a comparison of physiotherapist and orthopaedic surgeon recommendations for triage. J EvalClin Prac. 2009;15:178-183.

14. ACT Health. Expanding hospital services in the ACT and additional 400 Hospital Beds. Health Service Delivery, Public Consultation and Discussion Paper. Canberra: Australian Government; 2011. Available from: http://www.health.act.gov.au/consumer-information/communityconsultation/expanding-hospital-services-in-the-act/. Accessed June 22, 2011.

15. Australian Health Workforce Advisory Committee. The Australian Allied Health Workforce. An overview of Workforce Planning Issues, Report 2006.1. North Sydney: AHWAC NAHAC Endorsed National Definitions; 2006. Available from: http://www.ahwo.gov.au/documents/ Publications/2006/The\%20 Australian\%20allied\%20health\%20workforce.pdf. Accessed June 22, 2011.

16. ACT Health. ACT Health General Enterprise Agreement 2010-2011. Canberra; Australian Government; 2011. Available from: http://www health.act.gov.au/c/health?a=sendfile\&ft=p\&fid=-1135200739\&sid=. Accessed June 22, 2011.

17. Davis AM, Kitchlu N, MacKay C, Palaganas M, Wong R. Models of care delivery for people with arthritis. Working Paper (10-04). Toronto: Arthritis Community Research and Evaluation Unit, Toronto Western Research Institute; 2011. Available from: http://www.modelsofcare.ca/ pdf/10-04.pdf. Accessed May 25, 2011.
Patient Related Outcome Measures

\section{Publish your work in this journal}

Patient Related Outcome Measures is an international, peer-reviewed, open access journal focusing on treatment outcomes specifically relevant to patients. All aspects of patient care are addressed within the journal and practitioners from all disciplines are invited to submit their work as well as healthcare researchers and patient support groups. Areas covered will

\section{Dovepress}

include: Quality of life scores; Patient satisfaction audits; Treatment outcomes that focus on the patient; Research into improving patient outcomes; Hypotheses of interventions to improve outcomes; Short communications that illustrate improved outcomes; Case reports or series that show an improved patient experience; Patient journey descriptions or research. 